\title{
Motivación y rendimiento académico del idioma inglés
}

\author{
Motivation and academic performance of the English language
}

\author{
YONY DALCI TOLEDO PAUCAR ${ }^{1}$ \\ dalcitoledo@yahoo.com \\ https://orcid.org/0000-0001-9677-7073 \\ Universidad Nacional Mayor de San Marcos, Perú
}

\section{RESUMEN:}

El inminente desafío que se plantea en estos tiempos de pandemia dentro de las instituciones educativas es sentir el impulso que coadyuve a mantenerse motivado para demostrar un buen rendimiento académico como estudiante del idioma inglés. Por ende, las situaciones económicas, los espacios donde se imparten las clases en forma presencial y hoy, en forma remota, han generado el objetivo de la presente investigación: conocer sobre la motivación y su relación con el rendimiento académico en estudiantes del Centro de Idiomas de la UNMSM. Para tal fin, la investigación tuvo un enfoque cuantitativo sustentada en un análisis descriptivo. Seguidamente se presentó la realidad problemática, los antecedentes y las teorías que guardan relación con la motivación y el rendimiento académico. Se usó un instrumento de 24 ítems a través de la escala de Likert. Se analizaron e interpretaron los datos llegando a la conclusión que los estudiantes sanmarquinos tienen relevancia en los niveles de motivación para el aprendizaje del idioma inglés y las notas obtenidas para un excelente rendimiento, también son primordiales el conocimiento previo, la capacidad intelectual y los estilos de aprendizaje.

\begin{abstract}
:
The imminent challenge that arises in these times of pandemic within educational institutions is to feel the impulse that helps to stay motivated to demonstrate good academic performance as an English language learner. Therefore, the economic situations, the spaces where classes are taught in person and today, remotely, have generated the objective of this research: to know about motivation and its relationship with academic performance in students of the Language Center of the UNMSM. To this end, the research had a quantitative approach supported by a descriptive analysis. Then the problematic reality, the antecedents and the theories related to motivation and academic performance were presented. A 24-item instrument was used through the Likert scale. The data were analyzed and interpreted, reaching the conclusion that San Marcos students have relevance in the levels of motivation for learning the English language and the marks obtained for excellent performance, prior knowledge, intellectual capacity and styles of learning are also essential. learning.
\end{abstract}

\section{Palabras Clave:}

Motivación intrínseca; motivación extrínseca; rendimiento académico; motivación académica; consecuencias cognitivas.

\section{KEYWORDS:}

Intrinsic motivation; extrinsic motivation; performance academic; academic motivation; cognitive consequences.

Recibido: 30/08/2021 - Aceptado: 10/10/2021 - Publicado: 23/12/2021

\footnotetext{
(C) Los autores. Este artículo es publicado por la Revista peruana de investigación e innovación educativa de la Facultad de Educación, Universidad Nacional Mayor de San Marcos. Este es un artículo de acceso abierto, distribuido bajo los términos de la licencia Creative Commons Atribución 4.0 Internacional (CC BY 4.0) [https://creativecommons.org/licenses/by/4.0/deed.es] que permite el uso, distribución y reproducción en cualquier medio, siempre que la obra original sea debidamente citada de su fuente original.
} 


\section{Introducción}

La motivación se prioriza en casi todas las entidades universitarias que enseñan el inglés en especial en los institutos de idiomas de las universidades y es aplicado en los procesos de enseñanza universitarios, para ello se necesita herramientas vinculadas al proceso de motivación, para lograr el acceso a becas y artículos científicos que hoy en día benefician a los estudiantes universitarios para avanzar intelectualmente. Por ello Badillo (2020) indica que el idioma inglés se posesiona como el primer idioma usado por los investigadores y científicos del mundo entero, es decir, el 95\% de los artículos científicos son elaborados en inglés y un 1\% en portugués y español.

El avance exponencial tecnológico y digital en el mundo global, a través de la investigación, desarrollo e innovación, trae consigo lo valioso que es la enseñanza del inglés, para competir y obtener un puesto laboral donde los ingresos sean significativos y logren económicamente y socialmente un estatus en nuestra sociedad moderna, tomando relevancia la motivación en la práctica del inglés para los universitarios ylograr un adecuado nivel de rendimiento académico en su estadía en la Universidad.

Por lo tanto, se tiene un desafío latente dentro de las aulas donde se enseña el idioma inglés de manera presencial y no presencial; en ese contexto, se parte reconociendo que la motivación es un factor que guarda implicancia dentro de la conducta humana por ello es necesario que cada docente y estudiante conozca las características de su aplicación dentro de un contexto educativo y la asociación que tiene con el rendimiento universitario.

A continuación, tenemos distintos estudios precedentes, dentro de los mismos tenemos a Guillén et al. (2013) quienes sostienen que el gradode motivación se ve reflejada en las notas del curso de lengua extranjera, tomando en cuenta la motivación y el optimismo disposicional con el rendimiento académico en universitarios que se inician en el idioma inglés.

Lozano et al. (2000) indican que la motivación entorno al estudio de los idiomas extranjeros, contribuye productivamente a justificar el nivel del idioma francés e inglés y que el horizonte motivacional para educarse en el idioma extranjero se da en razón a las notas, un grupo tiene que tener una motivación intermedia necesaria para obtenerun excelente rendimiento.

Tejedor (2003) estudia el dominio demostrativo de ciertas condiciones en el rendimiento en los universitarios, relaciona varias variables, entre las más relevantes tenemos a la situación familiar, notas previas, motivación por los estudios, hábitos de estudio y satisfacción con la situación académica. Concluye que el intelecto, los procedimientos cognitivos nacen y se desarrollan en el entorno socio familiar y escolar donde interactúa el estudiante, determinando el rendimiento académico.

Tonconi (2010) realiza un análisis del rendimiento universitario y la deserción universitaria. Concluye que los estudiantes de gran rendimiento académico, con una elevada instrucción, terminan eficientemente sus estudios y los de menor instrucción no, y el reflejo directo en el rendimiento universitario son los elementos constitutivos como el grado de educación del principal agente familiar, la dedicación al estudio, la cantidad de cursos a llevar, la puntualidad en clase, los ingresos familiares mensuales yla cantidad de individuos en el hogar.

García (2012) investiga la correspondencia entre la motivación, la autoestima y elorden de calificaciones de 124 universitarios ingresantes de las diferentes escuelas. La investigación fue de tipo descriptiva, correlacional y transeccional. Finaliza mencionando que los universitarios con mayores progresos tienen una motivación individual y especialmente tener niveles altos de motivación intrínseca. Por lo tanto, tenemos una correlación relevante entre las variables estudiadas.

El concepto de motivación experimenta cambios y los diversos conceptos se parecen, según Reeve (2010) la motivación depende de la calidad tanto de los docentes como de los estudiantes, los estudiantes progresan y 
desarrollan habilidades flexibles con un buen producto y conceptos modernos, reflejándose en una autoestima superior apoyados por el docente que observa permisivamente el que hacer de los estudiantes. Además, Petri y Govern (2013) señalan que algunas veces queremos hacer algo y otras veces no, y que a menudo hablamos subjetivamente de nuestros motivos y lo expresamos como deseos, pulsiones y necesidades.

En cuanto a la motivación intrínseca, Schunk (2012) menciona que la tarea personal es importante en un acto con motivación intrínseca y que las retribuciones serán el triunfo individual en la tarea realizada satisfactoriamente aunado a una sensación competitiva.

Por otra parte, Ryan y Deci (2006) sobre las consecuencias cognitivas afirman que las semejanzas entre las diferentes motivaciones de los estudiantes, benefician al que esta motivado intrínsecamente en los distintos procedimientos como la retención y el amplio estudio de conceptos no frecuentes. Sobre las consecuencias afectivas, nos indican que los estudiantes en las aulas con docentes incisivos en controlar sus actividades, poseen sensaciones negativas y gozan de baja autoestima, empero estudiantes de clases con docentes más autónomos tienen más afectos positivos, son entusiastas, optimistas, curiosos y con mayor interés. Referente a las consecuencias motivacionales, infieren que la alegría y el propósito son superiores en la motivación extrínseca. Además, en las consecuencias conductuales enfatizan que los estudiantes supervisados superficialmente efectúan un reducido desempeño.

Según Ryan y Deci (2020) indican que, en el entorno educativo, la motivación intrínseca de elevada calidad, está relacionada con mejores resultados que la motivación de baja calidad, en el caso de la motivación a través de recompensas extrínsecas. Por otro lado, Gajardo (2012) afirma que la motivación académica es factorpredictivo ventajoso del rendimiento, junto al auto concepto y estrés para el control emocional.

Respecto al rendimiento académico, Xu y Wu (2013) señalan que la motivación yel rendimiento tienen correspondencia entre sus distintas variables como; la conducta a varias actividades y la actitud cordial afectuosa hacia las responsabilidades que posee un nexo con la administración de actividades individuales y en grupo. Por consiguiente, el vínculo positivo con el interés alrededor de los deberes es beneficioso individualmente y perjudicial en el aula.

Además, Núñez et. al (2014) señalan que si el estudiante que emplea más el enfoque intenso de preparación y acepte la motivation intrínseca tendrá un importante rendimiento y si emplea menos aquel enfoque, su rendimiento será moderado aceptando. Por lo tanto, individualmente estará motivado y orientado a realizar más actividades con considerables estrategias para su autorregulación en el estudio. Adicionalmente, Palmer (2013) manifiesta que la base de datos que poseen las instituciones sobre los estudiantes es beneficioso para el pronóstico del rendimiento. Igualmente, para la detección de nuevas variables relevantes e intervenir en ciertos ámbitos donde existe elevada deserción y bajo rendimiento.

Según García y García (2013) el resultado de los universitarios en las aulas corresponde a la certificación coherente en su rendimiento a través de puntuaciones cuantitativas y cualitativas.

Para Vargas (2013) el universitario que rinde, tiene diversas características y varios tipos de conocimientos para conseguir una adecuada nota cuantitativa mediante el cumplimiento de tareas universitarias vinculadas a los cursos aprobados o desaprobados y el abandono o la culminación universitaria.

Agudo et. al, (2014) indagan el rendimiento en la enseñanza virtual y en línea, tanto presencial y no presencial, considerando los modelos de interacciones en los temas en línea. Concluyen que dichos modelos no repercuten en los estudios con ambientes virtuales.

Siguiendo a Yu y Jo (2014) en su investigación, indican que los elementos significativos observados como; la cantidad de horas destinado al estudio, la periodicidad en el intermedio del estudio en un Sistema de Administración del Aprendizaje (Learning Management System), la interrelación entre los estudiantes y la 
cantidad de descargas, inciden en el rendimiento estudiantil. Además, determinan queel estudiante rinde cuando desarrolla tareas en ambientes virtuales.

Tempelaar et. al, (2014) estudian el poder de pronosticar las condiciones para la enseñanza, las consecuencias de las estimaciones pedagógicas permanentes y diversas informaciones originados por el rendimiento estudiantil para ocasionar un feed back. Concluyen que las evaluaciones formativas asistidas por el ordenador probablemente pronostica y localiza a los estudiantes de rendimiento reducido, mientrasque la información de un sistema de gestión del aprendizaje básicos no predice sustancialmente el aprendizaje.

Enea y Attanasio (2015) examinan los logros obtenidos por universitarios italianos mediante la media de sus notas (rendimiento cualitativo) y los creditajes aprobados (rendimiento cuantitativo). Comprueban que universitarios procedentes de instituciones educativas que enseńanza ciencias, son más sobresalientes de aquellos estudiantes egresados de instituciones técnicas. Por consiguiente, la puntuación cuando terminan la secundaria es un pronóstico revelador y que las ganancias generadas por el núcleo familiar no afectan el rendimiento universitario.

\section{Método}

El método empleado en el estudio es hipotético deductivo, porque se examinó a los individuos de la investigación y se elaboró una hipótesis sobre ellos. Además, el método de estudio de las variables es cuantitativa y el diseño del estudio es descriptivo y correlacional, tipificado en el mundo académico (Mejía, 2013). Así mismo, se administróuna serie de preguntas validadas por expertos a los universitarios.

Referente al estudio de la población fue conformada por estudiantes del curso del idioma inglés en la UNMSM y la muestra intacta estuvo representada por 30 estudiantes del nivel básico, Se considero el muestreo probabilístico por conveniencia. Sobre los resultados fueron netamente cuantitativos.

\section{Resultados}

Interpretación: De la tabla 1, se obtiene que el 70\% (21) de los estudiantes disfruta mucho de aprender un idioma, según los datos.

Tabla 1

Resultados respecto a las consecuencias afectivas

\begin{tabular}{lcc}
\hline Disfrutas mucho de aprenderun idioma & Frecuencia & Porcentaje \\
\hline Algo verdadero & 9 & $30 \%$ \\
Muy verdadero & 8 & $27 \%$ \\
Absolutamente verdadero & 13 & $43 \%$ \\
Total & 30 & $100 \%$ \\
\hline
\end{tabular}

Nota: Cuestionarios aplicados.

Interpretación: De la tabla 2, se obtiene que el 73\% (22) de los estudiantes considera que el estudiar inglés no es aburrido, según los datos.

Tabla 2

Resultados respecto a las consecuencias afectivas

\begin{tabular}{lcc}
\hline Piensas que este idioma esmuy aburrido & Frecuencia & Porcentaje \\
\hline Nada verdadero & 22 & $73 \%$ \\
Algo verdadero & 8 & $27 \%$ \\
Total & 30 & $100 \%$ \\
\hline
\end{tabular}

Nota: Cuestionarios aplicados. 
Interpretación: De la tabla 3, se obtiene que el 66\% (20) de los estudiantes estásatisfecho con su actuación en la tarea, mientras que un número reducido el 7\% (2) de estudiantes manifiesta estar insatisfecho, según los datos.

Tabla 3

Resultados respecto a las consecuencias cognitivas

\begin{tabular}{lcc}
\hline Estas satisfecho con tuactuación en esta tarea & Frecuencia & Porcentaje \\
\hline Nada verdadero & 2 & $7 \%$ \\
Algo verdadero & 8 & $27 \%$ \\
Muy verdadero & 16 & $53 \%$ \\
Absolutamente verdadero & 4 & $13 \%$ \\
Total & 30 & $100 \%$ \\
\hline
\end{tabular}

Nota: Cuestionarios aplicados.

Interpretación: De la tabla 4, se obtiene que el 57\% (17) de los estudiantes siente que puede hacer esta actividad muy bien y pocos estudiantes $7 \%$ (2) sienten que no puede hacerlo, según los datos.

Tabla 4

Resultados respecto a las consecuencias cognitivas

\begin{tabular}{lcc}
\hline Esta actividad lo puedeshacer muy bien & Frecuencia & Porcentaje \\
\hline Nada verdadero & 2 & $7 \%$ \\
Algo verdadero & 11 & $36 \%$ \\
Muy verdadero & 15 & $50 \%$ \\
Absolutamente verdadero & 2 & $7 \%$ \\
Total & 30 & $100 \%$ \\
\hline
\end{tabular}

Nota: Cuestionarios aplicados.

Interpretación: De la tabla 5, se obtiene que el 74\% (22) de los estudiantes no se siente obligado en estudiar inglés y la minoría el 13\% (4) si se siente obligado, según los datos.

Tabla 5

Resultados respecto a las consecuencias motivacionales

\begin{tabular}{lcc}
\hline Piensas que este idioma esmuy aburrido & Frecuencia & Porcentaje \\
\hline Nada verdadero & 22 & $74 \%$ \\
Algo verdadero & 4 & $13 \%$ \\
Muy verdadero & 4 & $13 \%$ \\
Total & 30 & $100 \%$ \\
\hline
\end{tabular}

Nota: Cuestionarios aplicados.

Interpretación: De la tabla 6, se obtiene que el 67\% (20) de los estudiantes piensa queel estudio de inglés es absolutamente importante, según los datos.

Tabla 6

Resultados respecto a las consecuencias motivacionales

\begin{tabular}{lcc}
\hline $\begin{array}{l}\text { Piensas que la realización deesta actividad } \\
\text { es importante. }\end{array}$ & Frecuencia & Porcentaje \\
\hline Muy verdadero & 10 & $33 \%$ \\
Absolutamente verdadero & 20 & $67 \%$ \\
Total & 30 & $100 \%$ \\
\hline
\end{tabular}

Nota: Cuestionarios aplicados. 
Interpretación: De la tabla 7, se obtiene que el 80\% (24) de los estudiantes concentratoda su atención en estudiar inglés, según los datos.

Tabla 7

Resultados respecto a la motivación académica

\begin{tabular}{lcc}
\hline $\begin{array}{l}\text { Cuando estas realizando esta actividad, } \\
\text { concentrastoda tu atención en ella }\end{array}$ & Frecuencia & Porcentaje \\
\hline Nada verdadero & 1 & $3 \%$ \\
Algo verdadero & 5 & $17 \%$ \\
Muy verdadero & 14 & $47 \%$ \\
Absolutamente verdadero & 10 & $33 \%$ \\
Total & 30 & $100 \%$ \\
\hline
\end{tabular}

Nota: Cuestionarios aplicados.

Interpretación: De la tabla 8, se obtiene que el 97\% (29) de los estudiantes piensa quela realización de estudiar inglés le ayuda a cumplir sus metas, según los datos.

Tabla 8

Resultados respecto a la motivación académica

\begin{tabular}{lcc}
\hline $\begin{array}{l}\text { Piensas que la realización de esta actividad } \\
\text { puede ayudarte para cumplir tus metas }\end{array}$ & Frecuencia & Porcentaje \\
\hline Nada verdadero & 1 & $3 \%$ \\
Algo verdadero & 4 & $14 \%$ \\
Muy verdadero & 25 & $83 \%$ \\
Total & 30 & $100 \%$ \\
\hline
\end{tabular}

Nota: Cuestionarios aplicados.

Interpretación: De la tabla 9, se obtiene que el 70\% (20) de los estudiantes tienen calificaciones aprobatorias entre 15 y 17, y una cantidad menor 6\% (2) entre 12 y 13, según los datos.

Tabla 9

Resultados respecto al rendimiento académico

\begin{tabular}{ccc}
\hline Calificaciones & Frecuencia & Porcentaje \\
\hline 12 & 1 & $3 \%$ \\
13 & 1 & $3 \%$ \\
14 & 8 & $27 \%$ \\
15 & 7 & $24 \%$ \\
16 & 9 & $30 \%$ \\
17 & 4 & $13 \%$ \\
Total & 30 & $100 \%$ \\
\hline
\end{tabular}

Nota: Exámenes aplicados.

\section{Discusión}

Sobre los resultados obtenidos, se infiere respecto a las consecuencias afectivas que el $70 \%$ disfruta aprender el idioma inglés y la mayoría de estudiantes (73\%) piensa que el idioma inglés no es aburrido. Asimismo, en las consecuencias cognitivas más de la mitad $66 \%$ está satisfecho con las tareas en clases y un $57 \%$ puede realizar la actividad muy bien al estudiar el idioma inglés.

Además, respecto a las consecuencias motivacionales el $67 \%$ piensa que el estudio de inglés es absolutamente importante. Sobre la motivación académica el $80 \%$ concentra su atención en estudiar inglés y la mayoría 
de estudiantes (97\%) piensa que la realización de estudiar inglés le ayuda a cumplir sus metas. El 70\% de estudiantes universitarios tuvo un excelente rendimiento académico. Todos estos hallazgos nos ratifican los otros resultados de investigaciones y la actitud teórica de Guillén et al. (2013) sostiene que el grado de motivación se ve reflejada en las notas del curso de lengua extranjera, tomando en cuenta la motivación y el optimismo disposicional con el rendimiento académico en universitarios que se inician en el idioma inglés. Además, Lozano, et al. (2000) menciona que la motivación entorno al estudio de los idiomas extranjeros, contribuye productivamente a justificar el nivel del idioma francés e inglés y que el horizonte motivacional para educarse en el idioma extranjero se da en razón alas notas, un grupo tiene que tener una motivación intermedia necesaria para obtenerun excelente rendimiento.

\section{Conclusiones}

Se concluye que los estudiantes del Centro de Idiomas tienen altos niveles motivacionales para aprender el inglés, con ciertas características especiales como el afecto positivo, el optimismo, el interés y no se sienten obligados a estudiar, concentrándose al máximo en sus actividades y en el cumplimiento de sus metas para su desarrollo académico y profesional.

Además, en la entidad donde se imparte el idioma inglés el extraordinario rendimiento universitario está asociado a la motivación intrínseca y extrínseca que traenconsigo consecuencias afectivas positivas para aprender el inglés que está vinculado a la satisfacción personal y conductual, así mismo, al conocimiento previo, a la capacidad intelectual y a los estilos de aprendizaje. Por lo tanto, tenemos consecuencias motivacionales positivas para la búsqueda de alternativas laborales con altos sueldos y una motivación académica para la obtención de un grado académico.

\section{Referencias}

Agudo, A., Iglesias, S., Conde, M., \& Hernández, Á. (2014). ¿Can we predict success from log data in VLEs? Classification of interactions for learning analytics and their relation with performance in VLE-supported F2F and online learning. Computers in Human Behavior, 542-550.

Badillo, A. (2020). Informe sobre diversidad lingüística en la ciencia en Espańa, Portugaly América Latina. OEA, Instituto Elcano.

Enea, M., \& Attanasio, M. (2015). An association model for bivariate data with application to the analysis of university students' success. Journal of Applied Statistics, 1-12.

Gajardo, A. (2012). Caracterización del rendimiento escolar de niños y niñas mapuches: contextualizando la primera infancia. Tesis doctoral, Universidad de Valladolid, Valladolid.

García, T., \& García, J. (2013). Aplicación de cuestionarios online sobre el rendimiento académico. Revista de Informática Educativa y Medios Audiovisuales, 10(17), $1-7$.

García, E. M. (2012). Relación de los factores, autoestima, motivación, puntaje de ingreso en el rendimiento académico de los alumnos ingresantes 2010, a la Universidad Nacional Tumbes. Tumbes: Tesis Universidad Nacional de Tumbes

Guillén, F., Pérez-Luzardo, J., \& Arnaiz, P. (2013). Relación entre motivación y optimismo disposicional en aprendices de inglés como lengua extranjera en un contexto universitario. Revista de Educación, Extraordinario, 104-128.

Hernández, R., Fernández, C. \& Baptista, M. (Eds.) (2014). Metodología de la Investigación. México: Mc Graw-Hill.

Lozano, L. M., García-Cueto, E., \& Gallo, P. (2000). Relación entre motivación y aprendizaje (Vol. 12). Oviedo: Psicothema.

Mejía, E. (2013). Investigación científica en Educación. Lima: Editorial UNMSM- CEPREDIM.

Núńez, J., Tuero, E., Vallejo, G., Rosario, P., \& Valle, A. (2014). "Variables del estudiante, del profesor y del contexto en la predicción del rendimiento académico en Biología: análisis desde una perspectiva multinivel". Revista de Psicodidáctica, 19(1), 145-172. 
Palmer, S. (2013). "Modelling engineering student academic performance using academic analytics". International journal of engineering education, 132-138.

Petri, H. L., \& Govern, J. M. (2013). Motivation: theory, Research and application (6 ed.).

Belmot: Wadswort Cengagen Learning.

Reeve, J. (2010). Motivación y Emoción. México D.F: Editorial Mac Graw Hill.

Ryan, R. M., \& Deci, E. L. (2006). Self-regulation and the problem of human autonomy: Does psychology need choice, self-determination, ¿and will? Journal of Personality, 74, 1557-1585.

Ryan, R. M., \& Deci, E. L. (2020). Intrinsic and extrinsic motivation from a self- determination theory perspective: Definitions, theory, practices, and future directions. Contemporary Educational Psychology, 61 (2020), p. 101860.

Schunk, D. H. (2012). Teorías del Aprendizaje. Una perspectiva educativa (Sexta ed.).

México: Pearson Education.

Tejedor, F. J. (2003). Poder explicativo de algunos determinantes del rendimiento en losestudios universitarios. Revista española de Pedagogía, 61, 5-32.

Tempelaar, D., Rienties, B., \& Giesbers, B. (2014). Computer Assisted, Formative Assessment and Dispositional Learning Analytics in Learning Mathematics and Statistics. Communications in Computer and Information Science, 67-78.

Tonconi Quispe, J. (2010). Factores que influyen en el rendimiento académico y la deserción de los estudiantes de la facultad de ingeniería económica de la Universidad del Altiplano. Cuadernos de Educación y Desarrollo, 2(1).

Vargas, G. M. (2013). Factores asociados al rendimiento académico en estudiantes universitarios desde el nivel socioeconómico: Un estudio en la Universidad de Costa Rica. Revista Electrónica Educare, 17(3), 57-87.

Xu, J., \& Wu, H. (2013). "Self-Regulation of Homework Behavior: Homework Management at the Secondary School Level”. The Journal of Educational Research, 106(6), 1-13.

Yu, T., \& Jo, I. (2014). Educational Technology Approach toward Learning Analytics: Relationship between Student Online Behavior and Learning Performance in Higher Education. ACM International Conference Proceeding Series, 269-270.

\section{Notas al final}

1 Licenciada en Educación por la Universidad Nacional Mayor de San Marcos. Además, cuenta con estudios de maestría en Docencia Universitaria y doctorado en Educación en la Universidad Nacional Mayor de San Marcos. Asimismo, se desempeña como docente en la Institución Educativa Particular Nuestra Señora de Montserrat. 